\title{
Shape Memory of Elastic Capsules Under the Effect of Spontaneous Shape
}

\author{
Zhe Gou ${ }^{1}$, Feng Huang ${ }^{2}$, Xiaodong Ruan ${ }^{1, *}$ and $\mathrm{Xin} \mathrm{Fu}^{1}$ \\ ${ }^{1}$ State Key Laboratory of Fluid Power \& Mechatronic Systems, Zhejiang University, \\ Hangzhou 310025, P.R. China. \\ ${ }^{2}$ College of Metrology \& Measurement Engineering, China Jiliang University, Hangzhou, \\ P.R. China. \\ Communicated by Boo-Cheong Khoo
}

Received 22 March 2017; Accepted (in revised version) 31 July 2017

\begin{abstract}
Red blood cells can recover their resting shape after having been deformed by shear flow. Their rims are always formed by the same part of the membranes, and the cells are said to have shape memory. Modeled as two-dimensional elastic capsules, their recovery motion and shape memory is studied, mainly focused on the effect of the spontaneous shape. The fluid-structure interaction is modeled using immersed boundary method. Based on the simulations, the resting shapes of capsules are obtained and the area ratio of spontaneous shape is found to play an important role. After remove of shear flow, all capsules can recover their resting shapes, while only capsules with noncircular spontaneous shapes present shape memory. As the spontaneous shape approaches a circle but still noncircular, the capsule spends more time on recovery process. We consider how these capsules deform depending on the membrane bending energy, and find that the relaxation speed is positive correlated to the range of values of dimensionless bending energy. These results may help to identify different spontaneous shapes for capsules especially RBCs through future experiments.
\end{abstract}

\section{AMS subject classifications: 74F10}

Key words: Fluid-structure interaction, immersed boundary method, shape memory, spontaneous shape.

\section{Introduction}

The deformation of fluid-filled capsules and vesicles have been a subject of intense research for many years. Such a structure shares some similar properties with red blood

\footnotetext{
*Corresponding author. Email addresses: gouzhe@zju.edu.cn (Z. Gou), hf@cjlu.edu.cn (F. Huang),
} xdruan@zju.edu.cn (X. Ruan), xfu@zju.edu.cn (X. Fu) 
cells (RBCs) which are the primary constituent of blood. The flow-induced deformation of an elastic capsule is a fundamental study for further understanding in medical and industrial applications. For example, blood diseases like malaria and sickle cell anemia can stiffen the cell membranes, leading to vascular embolism and hemolysis. The study is also important in design of microcapsules for certain purpose. Experiments and numerical simulations have shown that a single capsule or cell exhibits primarily two types of motion in simple shear flow: tank-treading (TT) motion in which the membrane rotating around the interior fluid while the cell forms a constant angle with the flow direction, and tumbling (TB) motion that the cell rotates like a rigid body $[15,17,24]$. Further studies revealed the energy barrier for the transition between TT and TB motions [35,38]. The dynamics of capsules and RBCs in various flow conditions have also been observed by experiments or predicted by numerical methods $[1,11,14]$.

Contrary to the deformation of capsules, the recovery motion from deformed states to initial resting shapes draws less interests. Micropipette aspiration [13] and optical tweezing [10] experiments have been used to study the deformation and relaxation of RBC membranes, and the time course of shape recovery shows some behavior consistent with the exponential decay function characterization. For RBCs deformed by shear flow, the relaxation of cell membranes can also been described by the same exponential decay function [4]. The recovery of two-dimensional (2D) biconcave capsules has been predicted numerically, and a two-part recovery is characterized by a pair of exponential decay functions [19].

RBCs exhibit shape memory during the recovery motion subsequent to shear-induced deformation [16]. After the removal of shear flow, the cell can return to its resting shape, and the rim of the cell is always formed by the same part of the membrane. This phenomenon implies that material elements at different position have different natural states, corresponding to some energy minimum shape of the membrane. Similarly, the shear experiments of Dupire et al. [11] and the centrifuge experiments of Hoffman et al. [21] further indicate anisotropic elastic properties of membrane. For RBCs both shear (spectrin network) and bending (lipid bilayer) elasticities might make a contribution to the deformation of the membranes. Thus shape memory could result from spatial inhomogeneous in either the natural state for shear elasticity or for bending elasticity or both.

So far, the main idea for the anisotropy of cell membrane comes from the stress-free state of spectrin network $[16,18]$. The stress-free state can affect cell dynamics in shear flow and it seems that the stress-free state of $\operatorname{RBC}$ is close to a spherical shape $[7,28,29]$. However, these arguments do not exclude the effect of bending rigidity. The bending energy of membrane is due to the curvature of lipid bilayer, and the spontaneous curvature is imposed to describe the chemical difference between the two monolayers [20]. In the following paragraphs, the term spontaneous shape corresponds to the shape determined by the spontaneous curvature. For capsules with uniform spontaneous curvature shapes (circle and flat plate), it has been shown that bending stiffness cause highly deformed capsules to develop round caps [25,32]. For capsules with resting shapes as spontaneous shapes, the capsules' steady shapes in extension flow are more akin to their resting shapes 
with increasing bending stiffness [37], and the energy barrier for TB-to-TT transition is also increased [38,39]. The biconcave shape developed by Evans and Fung [12] has been adopted by many researchers as the spontaneous shape of $\operatorname{RBC}[2,8,9]$. Capsules and vesicles composed of lipid bilayer only can also possess shape memory due to biconcave spontaneous shape $[19,27]$.

In the above researches, the capsules' spontaneous shapes are uniform curvature shapes (circle and flat plate) and the resting shapes of capsules, and these researches mainly focused on the effect of bending modulus on capsule dynamics. Apart from these spontaneous shapes, Sinha and Graham [33] suggests that an oblate spheroidal shape with spatially varying curvature results in better agreement with experiments than either a spherical or a biconcave spontaneous shape. Nevertheless, the difference among these three spontaneous shapes is subtle. Further investigation is needed to find distinguishable mechanisms that can be easily tested through experiments. On the other hands, although shape memory can not be eliminated after shearing for hours [16], recent optical tweezing experiments have shown that it can be changed or erased using very high power tweezers or long stretching time [6]. The change in biochemical milieu might also affect the spontaneous shape. Thus more variety of spontaneous shapes should be considered to model capsules and cells in certain situations.

So far, there is no related research on the effect of spontaneous shape on shape memory. Different spontaneous shapes might change the recovery process of cells and capsules dramatically, e.g. the recovery time and shape memory property. These results can be used as a qualitative guidance in identifying spontaneous shape in future experiments. The purpose of the present work is to numerically study the shape memory of capsules, especially its dependence on different spontaneous shapes with 2D models.

The rest of the paper is organized as follows. We begin with an introduction of the elastic model and the numerical algorithm, followed by the validation of our numerical method with previous results. Then the resting shapes of biconcave capsules with different spontaneous shapes are conducted as references of shear-induced deformations. With these preparations, we follow the "go-and-stop" method described by Fischer [16] and shape memory is simulated after the abrupt stop of shear flow for various spontaneous shapes. A brief interpretation is proposed based on the membrane bending energy, followed by the conclusions.

\section{Models and numerical method}

\subsection{Model}

In this paper, the membrane of capsule is treated as a continuous elastic shell. For a $2 \mathrm{D}$ model, the membrane is a curve with principle stretch ratio, $\lambda=\mathrm{d} s / \mathrm{d} S$. The term $\mathrm{d} s$ is the length of an infinitesimal element after some deformation while $\mathrm{d} S$ represents the length of the same element before deformation. The shear elasticity of the capsule is modeled 
with the Skalak model [34] as

$$
T=\left(\lambda^{2}-1\right)\left[G+K \lambda^{2}\left(\lambda^{2}+1\right)\right],
$$

where $G$ is the shear modulus and $K$ is the area modulus. It has been shown that the results for the Skalak model reach an asymptotic value for $K / G \geq 10[3,41]$. In the present study, Hooke's law is also employed to benchmark the numerical method:

$$
T=G(\lambda-1)
$$

To introduce the effect of spatially varied spontaneous curvature, we follow the approach by Pozrikidis [32] and define a bending energy

$$
E=\frac{E_{B}}{2} \int_{0}^{L}\left[\kappa(s)-\kappa_{0}(s)\right]^{2} \mathrm{~d} s,
$$

where $\kappa(s)$ and $\kappa_{0}(s)$ are the instantaneous curvature and the spontaneous curvature at point $s$ of the membrane respectively, and $E_{B}$ is the bending modulus. The bending force $f_{b}$ to minimize this energy can then be calculated by

$$
f_{b}=E_{B} \frac{\mathrm{d}}{\mathrm{d} s}\left[\frac{\mathrm{d}\left(\kappa-\kappa_{0}\right)}{\mathrm{d} s} n\right]
$$

where $n$ is the unit normal vector outward to the membrane. The total membrane strain force density is the combination of the shear and bending elastic forces:

$$
\boldsymbol{F}=\frac{\mathrm{d}}{\mathrm{d} s}(T \boldsymbol{\tau})+f_{b}
$$

where $\tau$ refers to the unit tangent vector.

To quantify different spontaneous shapes, we define the reduced area $A_{0}=4 \pi \mathrm{A} / \mathrm{L}^{2}$ as ratio of the enclosed area $A$ of a spontaneous shape to the area of circle with the same perimeter $L$. Thus the reduced area for the circular spontaneous shape is 1 , and other three spontaneous shapes to be investigated are: (i) oblate ellipsoid with $A_{0}=0.95$ (OE 0.95), (ii) oblate ellipsoid with $A_{0}=0.28$ (OE 0.28) and (iii) biconcave discoid with $A_{0}=$ 0.46 , as showed in Fig. 1. The OE 0.95 shape is nearly circular while its curvature is spatially varied. The thickness of the OE 0.28 shape is the same as the length between the two dimples of the biconcave shape. The biconcave discoidal shape follows the equations developed by Evans and Fung [12]:

$$
x=a \sin (\theta), \quad y=\frac{a}{2}\left[0.207+2.003 \sin ^{2}(\theta)-1.123 \sin ^{4}(\theta)\right] \cos (\theta),
$$

where $a$ is the discoid radius of biconcave shape. 


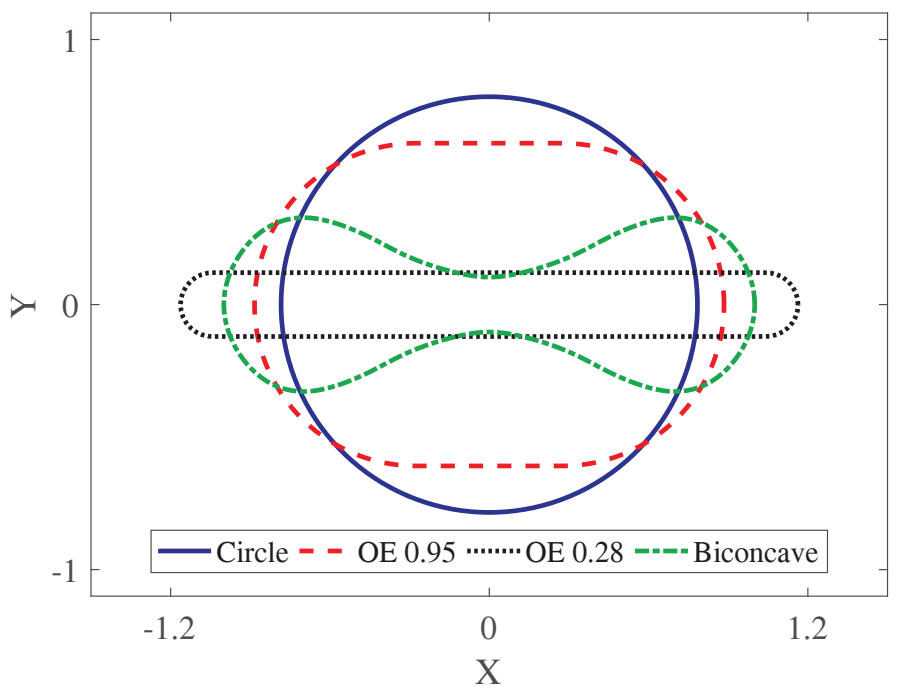

Figure 1: Contours of spontaneous shapes. The markers "OE 0.95 " and "OE 0.28 " represent oblate ellipsoid shape with reduced area of 0.95 and 0.28 , respectively.

\subsection{Numerical method}

The present work is based on the immersed boundary method developed by Peskin [30]. As shown in Fig. 2, the membrane boundary $\Gamma$ is represented by a Lagrangian coordinate $s$ (usually the arc length of the boundary), while the fluid domain $\Omega$ is represented by an Eulerian coordinate $x$. Then any point on the membrane has a corresponding Eulerian expression as $\boldsymbol{X}(s, t)$, where $t$ is time.

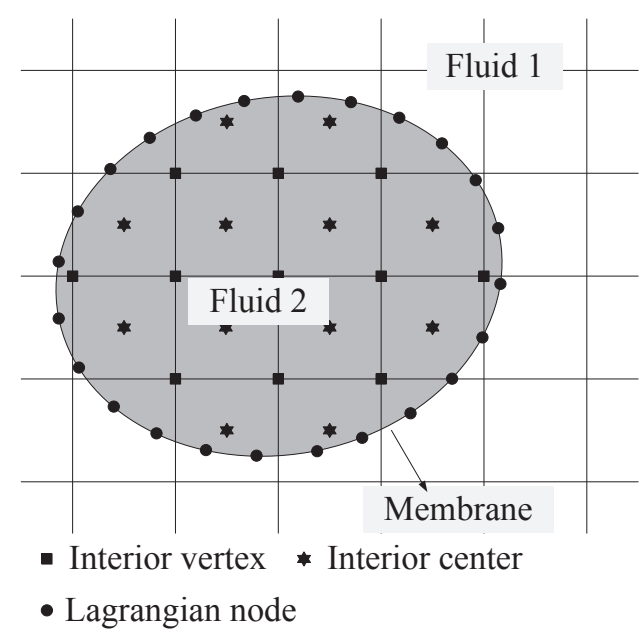

Figure 2: Schematic of a capsule immersed in fluids. The fluid domain is represented by a fixed Eulerian mesh. The membrane is represented by a moving Lagrangian mesh. 
For each Lagrangian point at $X(s, t)$, the membrane force spreads to the fluid as the force density $f(x, t)$ through a distribution function:

$$
f(x, t)=\int_{\Gamma} \boldsymbol{F}(s, t) \delta(x-X(s, t)) \mathrm{d} s .
$$

In the above equation, $\delta(\boldsymbol{x})$ is the Dirac delta function. Then the fluid flow is solved with finite volume method. Using the no-slip condition at each point of the membrane, the deformation of the membrane can be interpolated from the velocity field of the fluid:

$$
\frac{\partial}{\partial t} \boldsymbol{X}(s, t)=\boldsymbol{U}(\boldsymbol{X}, t)=\int_{\Omega} \boldsymbol{u}(\boldsymbol{x}, t) \delta(\boldsymbol{x}-\boldsymbol{X}(s, t)) \mathrm{d} \boldsymbol{x},
$$

where $\boldsymbol{U}(\boldsymbol{X}, t)$ is the velocity of the Lagrangian point at location $\boldsymbol{X}$.

In previous researches, the viscosity ratio was set to 1 for simplicity $[8,9,36-38]$. However, it has been proved that the capsule dynamics, e.g. the transition from tank-treading to tumbling, is related to viscosity ratio across the membrane $[18,22,33]$. The latticeBoltzmann method uses a viscosity function to describe lattice points inside and outside the membrane at any given time [23]. Other investigators use boundary element method to solve the Stokes equations $[22,25,32,33]$, and the viscosity ratio is explicitly contained in this algorithm. Some mesh-free approach like dissipative particle dynamics [42] and smooth particle hydrodynamics [31] denote the interior and exterior fluids with different particles. For the finite volume method combined with immersed boundary method, a resulting Poisson equation is solved to track the moving boundary in the fluid domain [40]. This algorithm is time consuming and inconvenient for parallel solution. In the present work, we follow the cell center and vertex flagging method produced by Blais et al. [5], which is highly parallelizable. For each cell $i$ of the fixed Eulerian mesh, the numbers of cell vertices $N_{v c, i}$ and cell center $N_{c c, i}$ inside the membrane are counted, as illustrated in Fig. 2. The interior volume fraction $\beta_{i}$ is generated by following equation:

$$
\beta_{i}=\frac{N_{v c, i}+N_{c c, i} N_{v, i}}{2 N_{v, i}}
$$

where $N_{v, i}$ is the number of vertices of cell $i$ (i.e. 4 for a square grid). With this volume fraction function $\beta_{i}$, the viscosity of cell $i$ is defined as

$$
\mu_{i}=\left(1-\beta_{i}\right) \mu_{e x}+\beta_{i} \mu_{i n}
$$

where $\mu_{\text {in }}$ is the interior viscosity, and $\mu_{\text {ex }}$ the exterior viscosity.

\subsection{Validation}

The deformation of initially circular capsules is studied to validate the present numerical method. The membrane includes shear elasticity with Hooke's law and bending stiffness with the spontaneous shape the same as the initial circular shape. The viscosity jump 
(a)

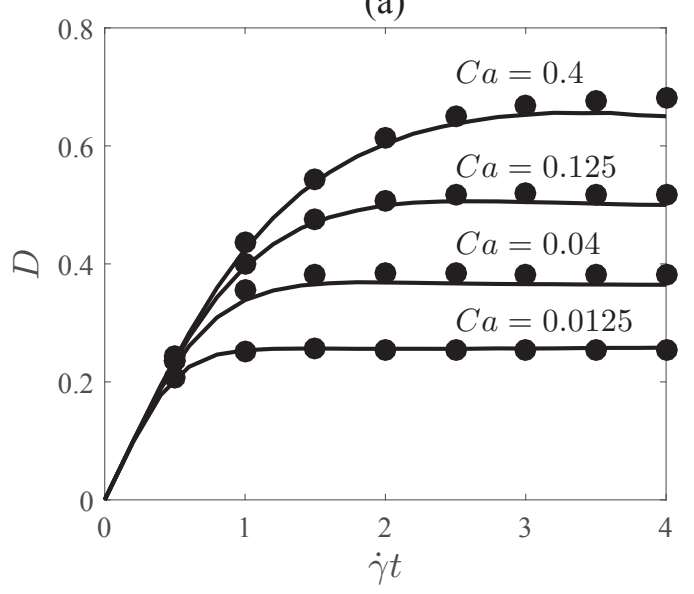

(b)

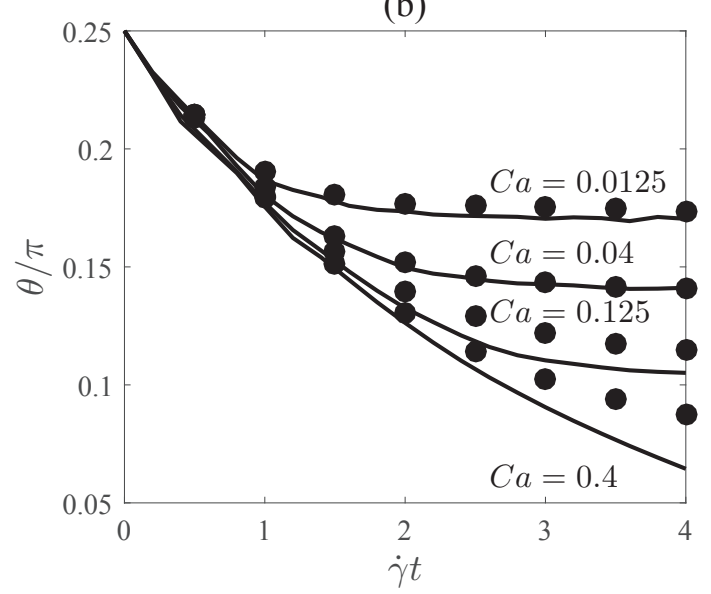

Figure 3: Time variation of (a) Taylor deformation parameter and (b) inclination angle for $e_{B}=0$. The solid lines represent the results of present method and dots are data points from [36].

cross the membrane is not considered. Two dimensionless parameters are identified to play an essential role in determining the capsule deformation. The dimensionless shear modulus is expressed by the capillary number $C a=\mu_{e x} \dot{\gamma} a / G$, where $\dot{\gamma}$ is the shear rate. The bending modulus is expressed nondimensional as $e_{B}=E_{B} / a^{2} G$. To quantify the deformation of capsules, the Taylor deformation parameter $D$ and inclination angle $\theta$ are introduced. The Taylor deformation parameter is defined as

$$
D=\frac{L-B}{L+B}
$$

where $L$ and $B$ are the major and minor axes of capsule. The inclination angle $\theta$ refers to the angle between the major axis of capsule and the horizontal direction of flow, in the range of $\left[-\frac{\pi}{2}, \frac{\pi}{2}\right]$.

The time variation of the capsule deformation parameter $D$ and inclination angle $\theta$ are compared with previous results of Sui and coworkers $[36,38]$ by lattice-Boltzmann method combined with the immersed boundary method. In Fig. 3 the effect of different shear modulus in the absence of bending resistance is shown, and good quantitative agreements are observed. Moreover, the effect of bending modulus is considered with fixed capillary number $\mathrm{Ca}=0.04$ in Fig. 4 . The present results match quite well with previous data.

\subsection{Set up}

In the present study, the discoid radius $a$ is chosen as the characteristic length and $1 / \dot{\gamma}$ the characteristic time. The Reynolds number is kept less than 0.001 to achieve the Stokes flow condition. The exterior viscosity is varied while other parameters are kept constant 
(a)

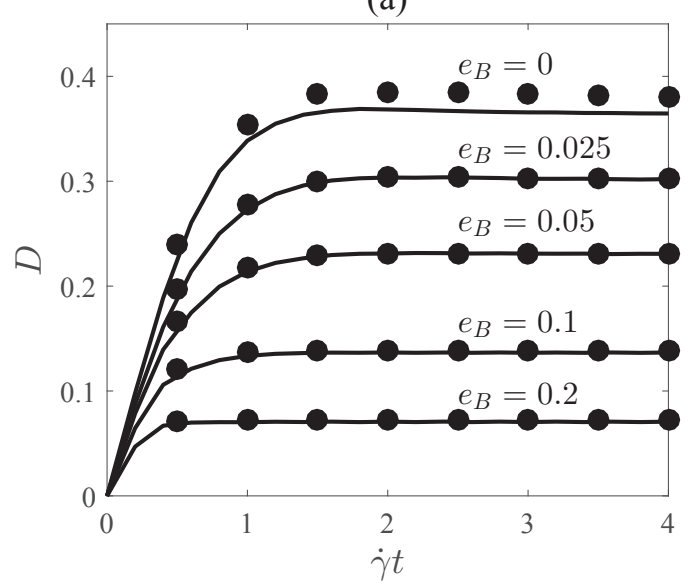

(b)

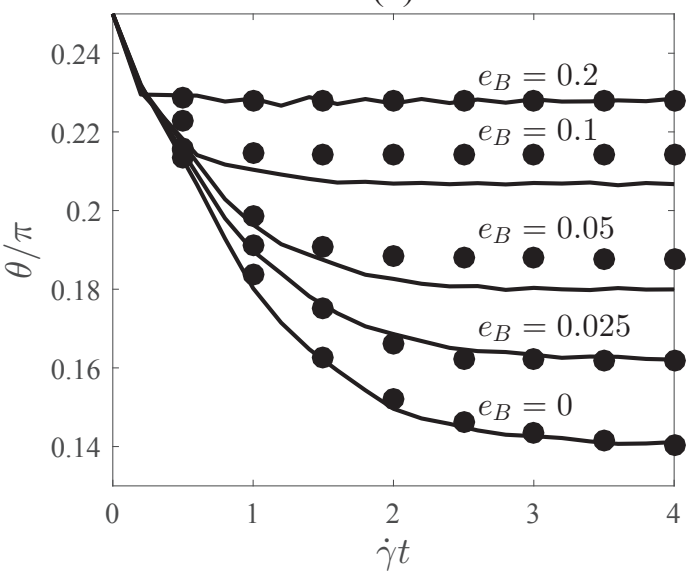

Figure 4: Time variation of (a) Taylor deformation parameter and (b) inclination angle for $\mathrm{Ca}=0.04$. The solid lines represent the results of present method and dots are data points from [38].

so that different capillary numbers are obtained. The computational domain is a $5 a \times 5 a$ square, with 101 nodes in each direction. A capsule with an initial biconcave shape is placed in the center of the computational domain. The capsule membrane is divided into 200 discrete segments with equal length. The whole simulations are performed by the following steps:

1. The capsule is released in still fluid. If the spontaneous shape is differ from the initial biconcave shape, the capsule would deform from its initial shape, and finally reaching resting shape.

2. A linear shear flow is induced by translation movement of the top and bottom boundaries to the opposite direction. Then the capsule would perform TT motion, and the membrane would rotating around the interior fluid.

3. The shear flow is stopped when the material point forms the rim of the membrane at rest (hereafter referred to as rim point) is at the dimple of the capsule. The successive relaxation of capsule and the motion of the rim point are recorded.

\section{Results and discussion}

\subsection{Resting shapes}

Our research starts with the resting shapes of capsules in hydrostatic fluid. The final resting shapes for different spontaneous shapes and the pressure fields after being normalized by $G / a$ are shown in Fig. 5 . The solid lines represent the resting shapes and the dash lines represent the biconcave shapes. When the spontaneous shape is circle or 

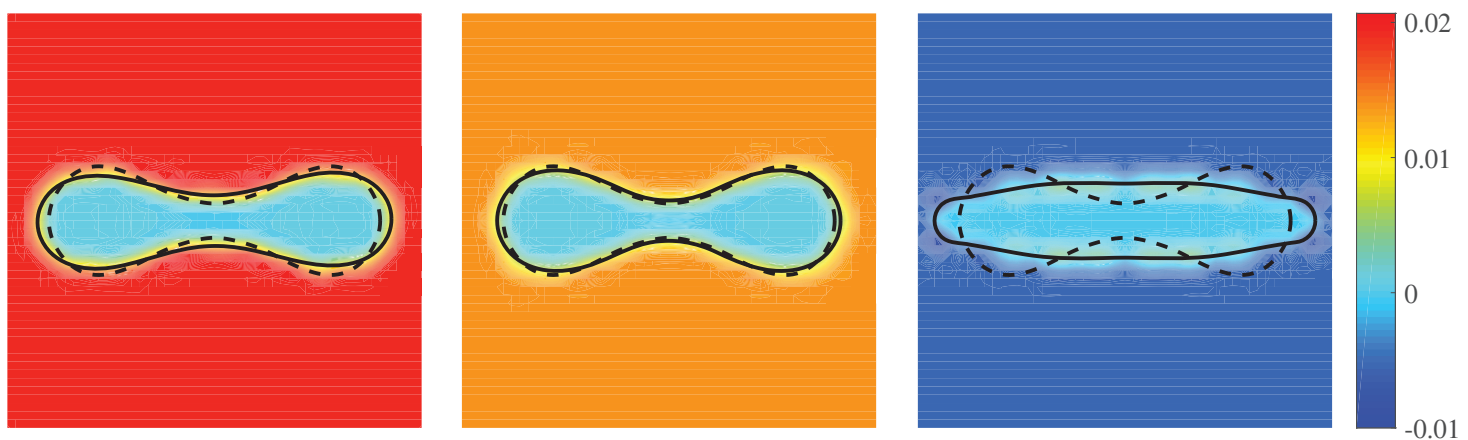

Figure 5: Normalized pressure contours of the resting shape with different spontaneous shapes: circle (left), OE 0.95 (middle), OE 0.28 (right). The solid lines represent the resting shapes and the dash lines represent the biconcave shapes.

OE 0.95 , the resting shape is still a biconcave discoid with a slightly shallower dimple. Apparently the resting shape of the OE 0.95 capsule is closer to the biconcave capsule. However, when the spontaneous shape is OE 0.28 , the dimple disappears and the resting shape is more like an ellipsoid other than a biconcave discoid. This is reasonable for its reduced area is smaller than the one of biconcave shape, leading to a swelling from the spontaneous shape. It should be noticed that resting shape is not affected by $\mathrm{Ca}$, since it only relates to spontaneous shape for $2 \mathrm{D}$ capsules. These resting shapes are chosen as references for the study of shape memory.

Explicit pressure jumps are observed across the membrane in all three cases with nonbiconcave spontaneous shape, indicating the residual stress of membrane at rest. Interestingly, the pressure differences between the interior and exterior fluid are negative for the circle and the OE 0.95 capsules while positive for the OE 0.28 capsule. As can be seen from Fig. 5, the pressure difference is affected by the spontaneous shape, mainly the reduced area $A_{0}$. With the decreasing of $A_{0}$, the absolute value of pressure difference first decreases and then increases. The pressure difference reaches 0 when $A_{0}=0.46$, which is the same as the reduced area of the biconcave capsule.

\subsection{Go-and-stop simulations}

In the go-and-stop experiments developed by Fischer [16], a RBC is first sheared to perform TT motion (go). Then the shear flow is stopped when the rim point is at the dimple of cell (stop). According to his research, the rim point can finally recover to the rim, and the cell is said to possess shape memory. Following simulations are based on this go-and-stop procedure, and the effect of spontaneous shapes is shown.

Fig. 6 shows deformations of capsules in go-and-stop simulations at $\mathrm{Ca}=0.086$. The blue lines denote the contours of capsules and the red dots represent the rim points. The numbers below each contour indicate dimensionless time $\dot{\gamma} t$. Shear flows are stopped at $\dot{\gamma} t=0$, and the negative numbers refer to TT motion of capsules. In the first column the 
(a)

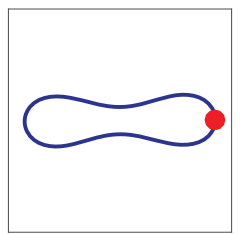

resting shape

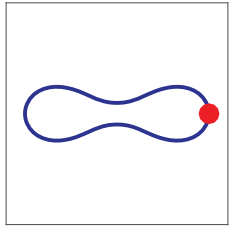

resting shape

(c)

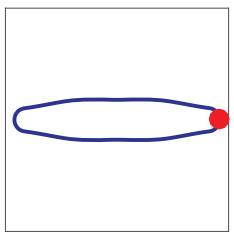

resting shape

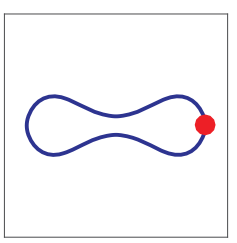

resting shape

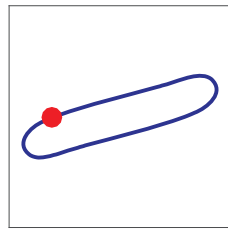

$-5$

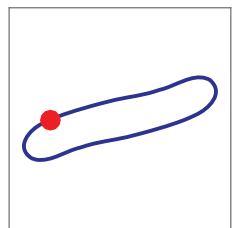

$-5$

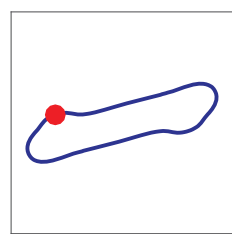

$-5$

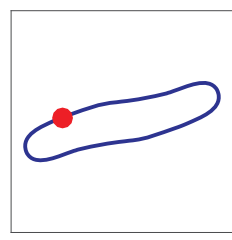

$-5$

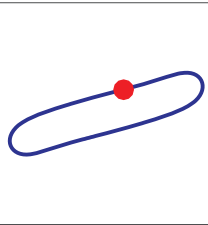

0

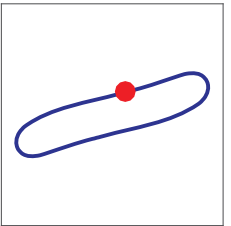

0

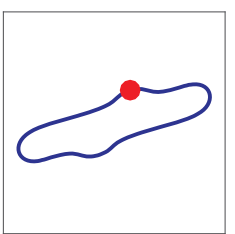

0

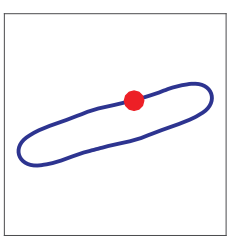

0

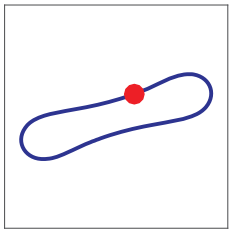

5

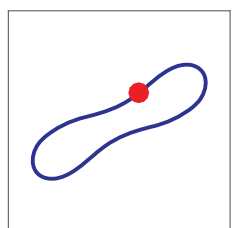

80

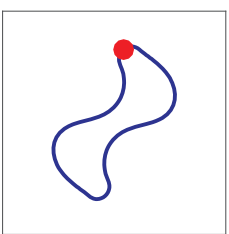

20

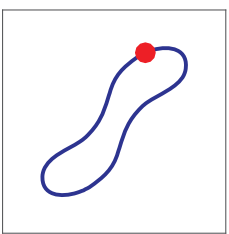

20

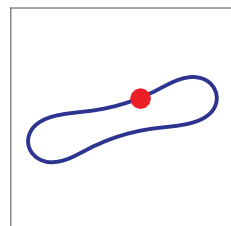

10

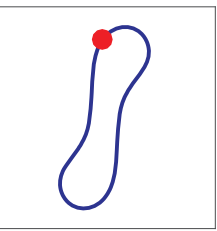

200

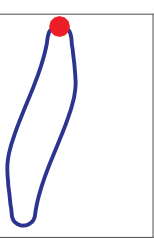

40

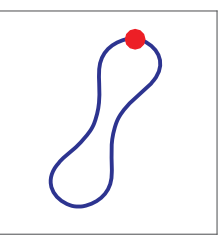

40

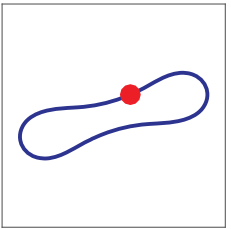

20

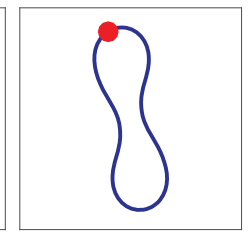

340

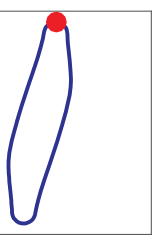

60

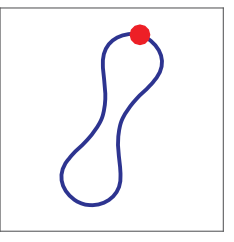

60

Figure 6: Go-and-stop simulations considering different spontaneous shapes: (a) circle, (b) OE 0.95, (c) OE 0.28 , and $(\mathrm{d})$ biconcave in shear flow at $C a=0.086$. The blue lines denote the contours of capsules and the red dots represent the rim points on the membrane. The first column shows resting shapes of capsules. The numbers indicate dimensionless time $\dot{\gamma} t$, and the flows are stopped at $\dot{\gamma} t=0$.

resting shapes are shown and the rim points are at the rims of capsules. From comparison between the second and third column we can see that all capsules perform TT motion with a swinging mode. The OE 0.28 cell has two protrusions rotating around interior fluid, due to the spatial variation of spontaneous shape. This phenomenon is analogous to the motion of capsules with elliptical resting shape described by Sui et al. [38].

After stop, the dimples become evident for all capsules in the beginning. These results are similar to the observation of Fischer [16] that the RBC first returns to a biconcave shape before tank treading. For the OE 0.28 capsule the dimple vanishes with time as the capsule recovering to resting shape. All capsules can recover to their resting shapes, while the motions of rim points divide them into two groups. For circle capsule in Fig. 6(a), the TT motion stops right after the shear flow stops and the rim point stays at the dimple till the capsule reaches resting shape. Capsules with noncircular sponta- 
neous shapes (Figs. 6(b-d)) perform a combination movement of TT and flipping after the shear flow stops. Their rim points return to the rims at final resting states, similar to the observation of Fischer [16]. It seems that the shape memory comes from noncircular spontaneous shape. The tank-treading of membrane exerts a torque on the cell that lead to a counter-clockwise flipping motion. This torque is also responsible for the inclination angle during the steady state TT motion in shear flow [24].

It is noteworthy that the OE 0.95 cell take longest time to recover to its resting shape, while the other two capsules with noncircular spontaneous shapes recover faster. As the OE 0.95 spontaneous shape approaches a circle, its curvature value varies in a relatively smaller range comparing to the other two capsules, resulting in smaller energy barrier for different configurations of the rim point. Thus we can hypothesize that for capsule with noncircular spontaneous shape, the smaller the range of spontaneous curvature is, the longer the recovery time is. Sinha and Graham [33] have reported similar results that the critical shear rate for TT-to-TB transition is smaller for spontaneous shape akin to sphere than biconcave shape, indicating larger energy barrier for the biconcave spontaneous shape. However, they did not consider the effect of spontaneous shape on the recovery motion of cell.

To quantify the recovery motion of capsules, the phase angle $\beta[26]$ is introduced as

$$
\beta(t)=\alpha(t)-\theta(t)-\left(\alpha_{0}-\theta_{0}\right),
$$

in which $\alpha(t)$ is the current angle between the membrane element and the direction of flow, $\theta(t)$ is the current inclination angle, and $\alpha_{0}$ and $\theta_{0}$ are the initial values of these angles. In the present work, the membrane element refers to the rim point, and the range of $\beta$ is $\left[0, \frac{\pi}{2}\right]$. Thus for a capsule in resting shape, the value of $\beta$ is 0 . Actually, the temporal evolution of phase angle $\beta$ reflects the movement of rim point. If the phase angle can return to 0 at resting shape, the capsule must possess shape memory. The variation of $\beta$ is shown in Figs. 7(a). For circle spontaneous shape, the phase angle is nearly constant, which means circle spontaneous shape is not related to shape memory. For OE 0.95 and biconcave capsules, as the $\beta$ values monotonically decrease to 0 , the rim points also return to the rims of capsules. For OE 0.28 capsule the $\beta$ value first increases and soon decreases to 0 . The increasing period does not mean an inversely TT motion, but might be related to the two protrusions as shown in Fig. 5(c).

The variation of inclination angle $\theta$ is shown in Figs. 7(b). The inclination angle for circle spontaneous shape is almost unchanged comparing to its initial value. For capsules with noncircular spontaneous shapes, the increasing of $\theta$ values indicates the flipping motions shown in Fig. 6.

The relaxation process in terms of Taylor deformation parameter $D$ for $C a=0.086$ is show in Figs. 8(a) and (b). For circle capsule, the value of $D$ first increases within a short period and then declines slowly. The $D$ value of biconcave capsule first decreases in a short time and then increases for a much longer period. This relaxation process with two separate modes follows the results of Gounley and Peng [19]. The curve trend for OE 0.28 capsules seems to be an inversion for biconcave capsule on horizontal axis, but the 
(a)

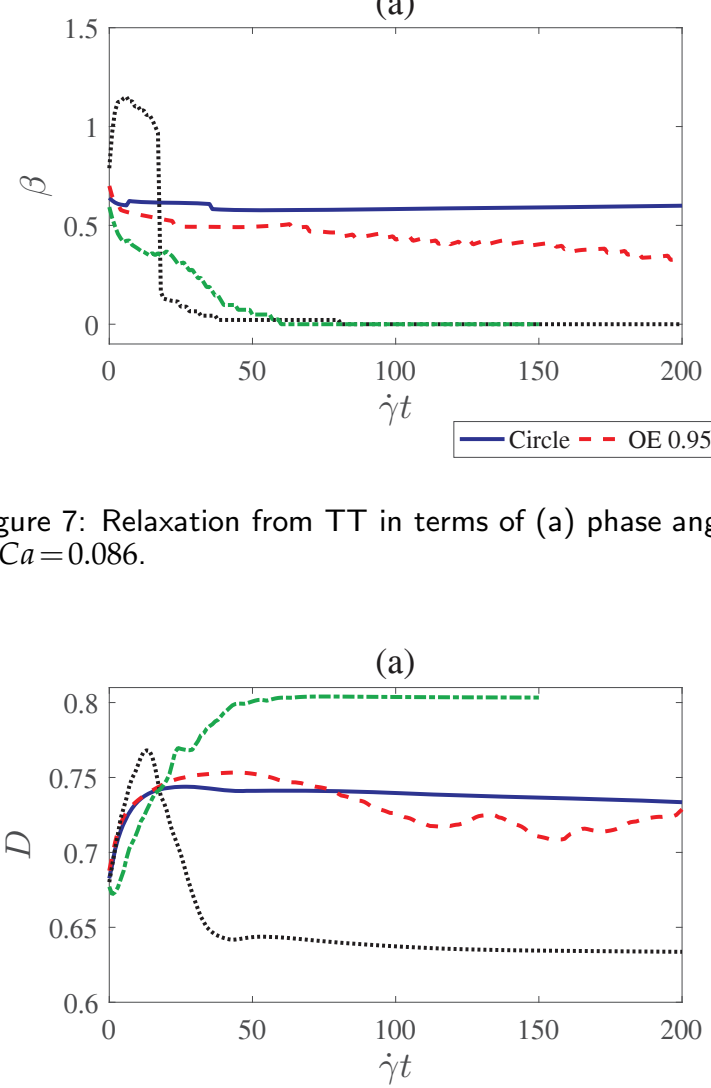

(c)

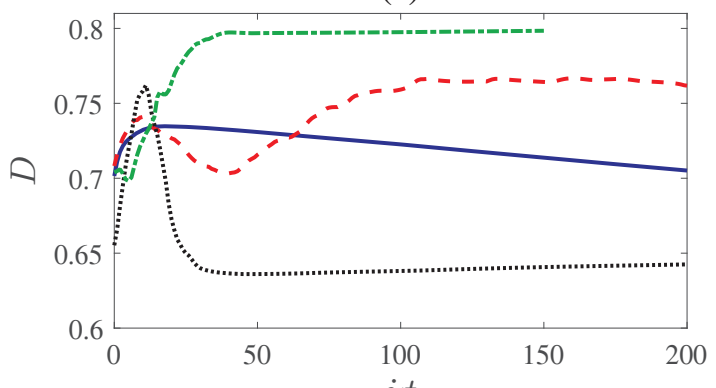
is $C a=0.086$ (b)

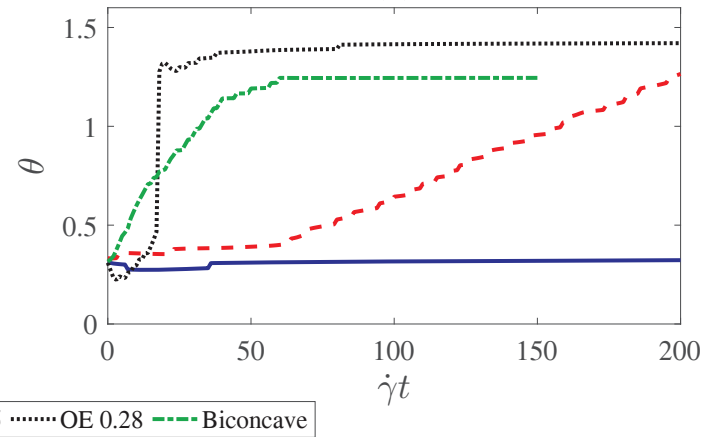

Figure 7: Relaxation from TT in terms of (a) phase angle $\beta$ and (b) inclination angle $\theta$. The capillary number

(b)

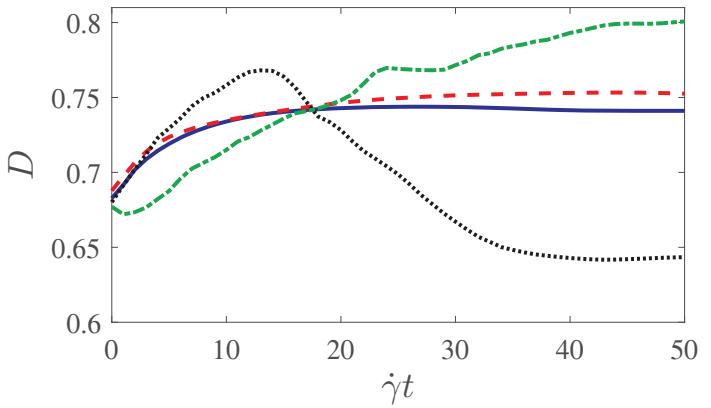

(d)

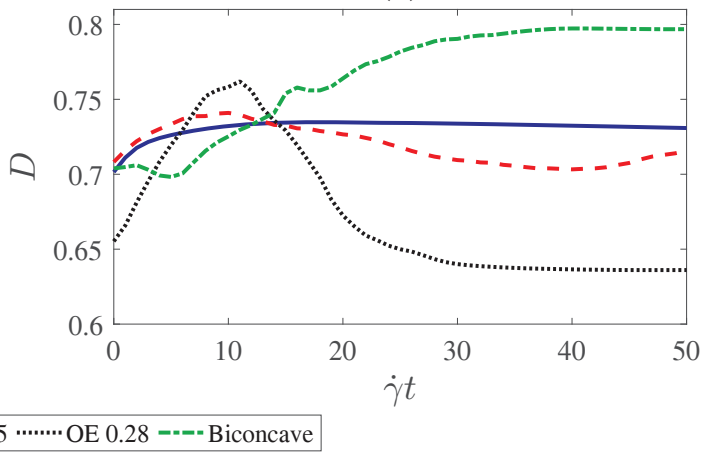

Figure 8: Relaxation from TT in terms of Taylor deformation parameter $D$. The capillary numbers are $C a=0.086$ (first row) and $\mathrm{Ca}=0.039$ (second row). The figures in right column enlarge the horizontal axes of figures in left column.

turning point arises later. The case for OE 0.95 capsule is the most complicated among others. In the first period the $D$ value rises with a speed nearly the same as the circle capsule. Then the first turning point shows up and the value decreases slowly. After a 
fluctuation period around $\dot{\gamma} t=150$, the value increases again until the capsule reaches resting shape (not shown in Fig. 8(a)).

Figs. 8(c) and (d) refer to the variation of $D$ at $C a=0.039$. The turning points for corresponding curves appear earlier when compared with Figs. 8(a) and (b). The comparison of top and bottom rows in Fig. 8 shows similar relaxation processes, but the relaxation time is shorter for less $\mathrm{Ca}$ value. The relaxation of capsule reflects the dissipation of membrane energy in viscous fluids, which is dominated by the elasticity and bending stiffness of the membrane, along with dissipation in the fluid. In the above simulations, the change of $\mathrm{Ca}$ comes from different $\mu_{e x}$ values while other parameters are kept constant. Thus higher $\mathrm{Ca}$ means larger exterior viscosity, bringing more resistance to the relaxation process of capsule.

\subsection{Bending energy}

The bending modulus $E_{B}$ is chosen as the energy scale, and the variation of nondimensional membrane bending energy $E / E_{B}$ is demonstrated in Fig. 9. At $C a=0.086$ the bending energies decrease in two modes: initial quick decreasing mode and later slow decreasing mode, separated by obvious turning points (Fig. 9(b)). While at $\mathrm{C} a=0.039$ the bending energies seem to follow the later mode at $C a=0.086$. For capsules in shear flow, spontaneous shapes increase the energy barrier for TB-to-TT transition. More energy is stored in the membrane when $\mathrm{Ca}$ is higher. During the relaxation process, the motion of capsule is driven by extra energy exceeding this energy barrier. It seems that there exists a critical energy value. For bending energy higher than this critical value, the dissipation follows two modes like Figs. 9(a) and (b). Otherwise the dissipation only has one mode.

The bending energies for OE 0.95 capsules take much longer time to decrease to equilibrium values than other two capsules with noncircular spontaneous shapes. Fig. 6 and Fig. 7(a) show analogous phenomenon. To further explain this phenomenon, we defined a dimensionless bending energy density as

$$
\omega(g)=\frac{e_{t}(g)-e_{\infty}(g)}{E_{\infty}} \cdot L \cdot \frac{\mu_{e x}}{\mu_{i n}}
$$

where $g$ is the dimensionless curve length varying from 0 to 1 as $s$ varies from 0 to $L, E_{\infty}$ is the bending energy of the resting shape, and $e_{t}$ and $e_{\infty}$ are bending energy densities at dimensionless time $\dot{\gamma} t$ and resting shape respectively. The bending energy density is defined as

$$
e(g)=\frac{E_{B}}{2}\left[\kappa(g)-\kappa_{0}(g)\right]^{2}
$$

As mentioned above, $E_{\infty}$ and $e_{\infty}$ refer to the energy barrier induced by spontaneous shapes, and $e_{t}-e_{\infty}$ corresponds to the extra energy exceeding energy barrier. Thus the $\omega$ value is 0 for any segment of the membrane when the capsule recovers to resting shape. According to the above definition, the value of $E_{\infty}$ is 0 for the biconcave capsule, which leads to an infinite value of $\omega$. Thus biconcave capsule is not considered in the following 

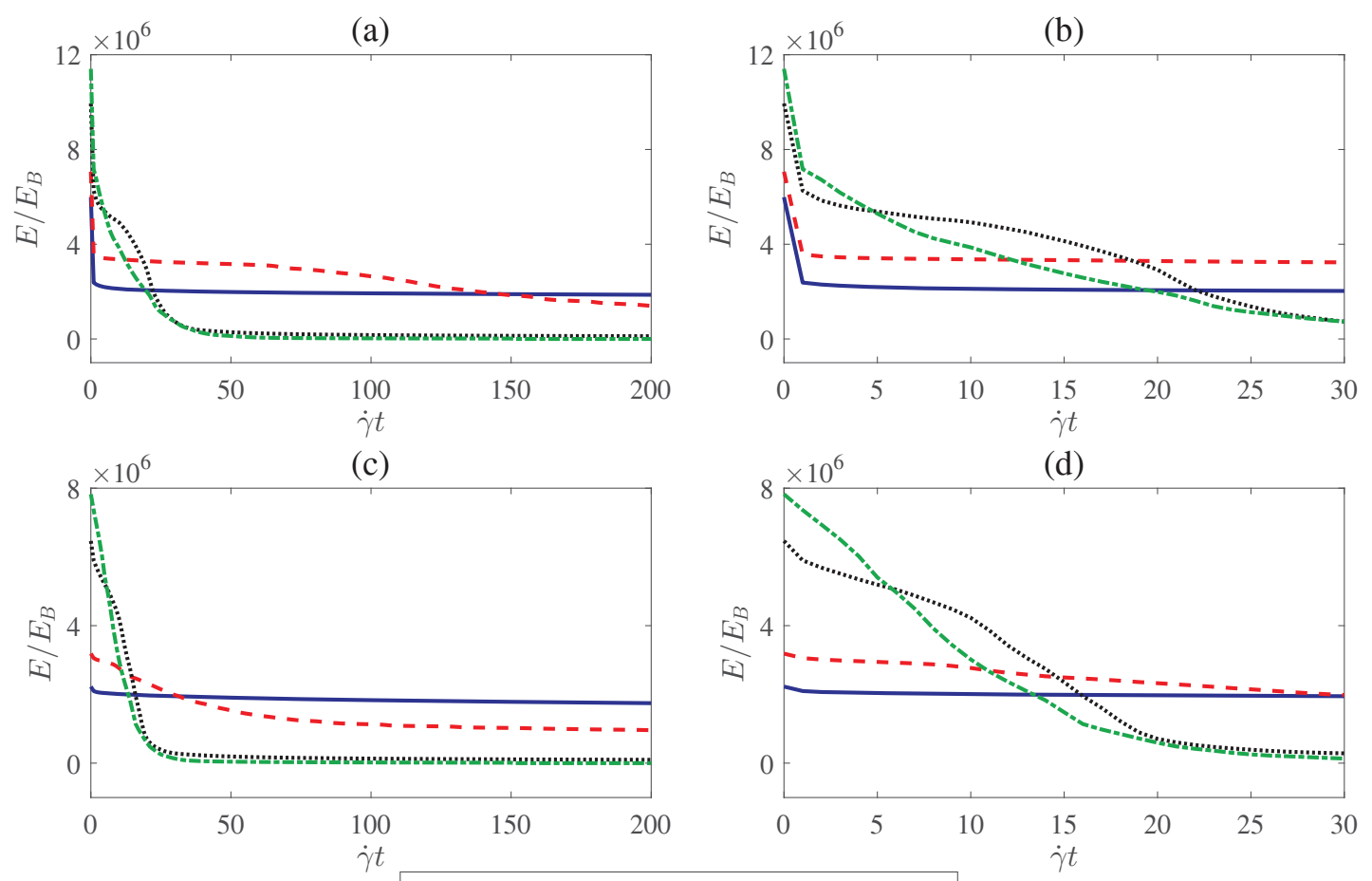

—Circle - - OE 0.95 …… OE 0.28 ---- Biconcave

Figure 9: Time variation of the membrane bending energy in terms of different capillary numbers: $C a=0.086$ (first row) and $\mathrm{Ca}=0.039$ (second row). The figures in right column enlarge the horizontal axes of figures in left column.

(a)

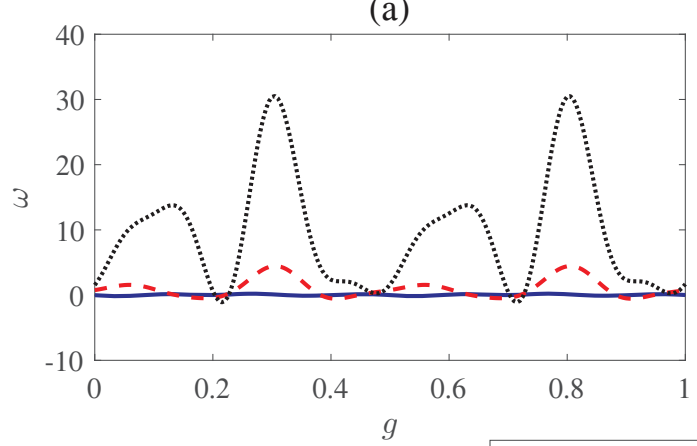

(b)

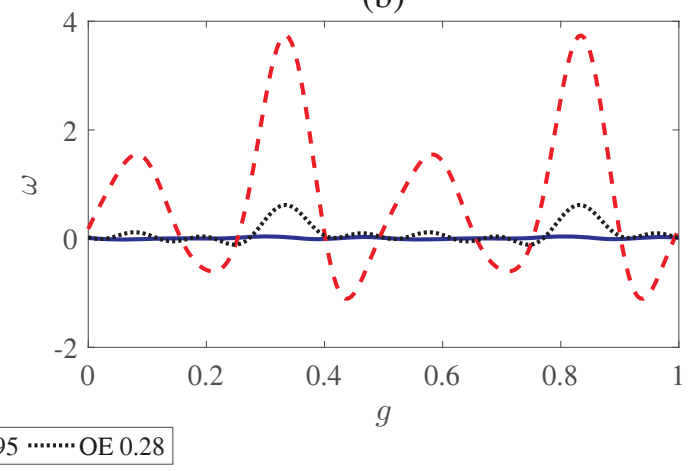

Figure 10: Variation of dimensionless bending energy $\omega$ with $g$ at (a) $\dot{\gamma} t=20$ and (b) $\dot{\gamma} t=100$. The capillary numbers is $C a=0.086$.

discussion. Fig. 10 shows the variation of $\omega$ with $g$ at $\dot{\gamma} t=20$ and $\dot{\gamma} t=100$. At $\dot{\gamma} t=20$, the OE 0.28 capsule has the largest amplitude of $\omega$. While at $\dot{\gamma} t=100$, the $\omega$ amplitude of OE 0.95 capsule becomes the largest. 


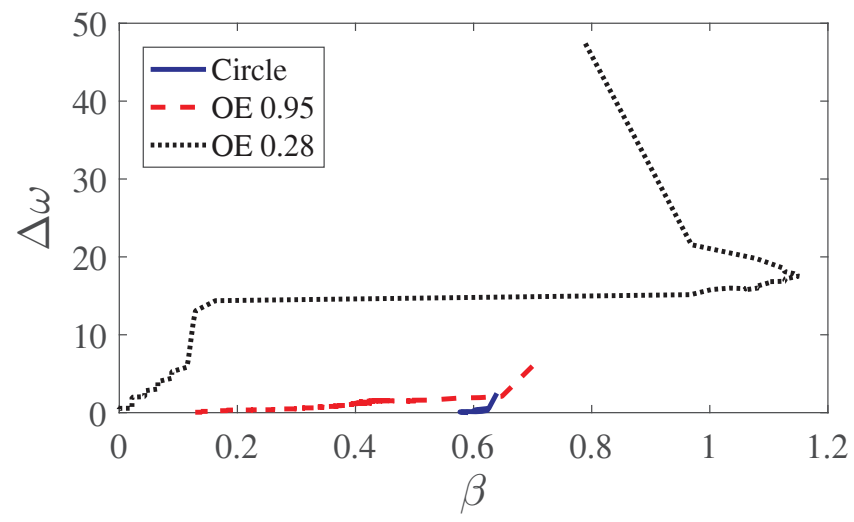

Figure 11: Variation of $\Delta \omega$ with dimensionless time at $C a=0.086$.

The amplitude of $\omega$ is defined as

$$
\Delta \omega=\max (\omega)-\min (\omega),
$$

where $\max (\omega)$ and $\min (\omega)$ refer to the maximum and minimum value of $\omega$ at given time. Variation of $\Delta \omega$ with $\beta$ is shown in Fig. 11. For capsules with same shape and same $\beta, \Delta \omega$ is affected by the range of spontaneous curvature. The value of $\Delta \omega$ is also 0 at resting shape. The $\Delta \omega$ value for OE 0.95 capsule remains smaller than the one for OE 0.28 capsule at any $\beta$ value. It seems that the TT speed in relaxation process is positive correlated with the range of dimensionless bending energy density. For capsule at same phase angle, spontaneous shape with large reduced ratio would have small range of spontaneous curvature, leading to small $\Delta \omega$ value and long relaxation time. The value of $\Delta \omega$ for circle capsule is even smaller than the one for OE 0.95 capsule, but the isotropic spontaneous curvature greatly reduce the energy difference of resting shape at different $\beta$. Hence the circle capsule performs no TT during relaxation process, leading to rapid relaxation.

\subsection{Phase angle differences}

It has been shown that the relaxation of capsule is related to the phase angle when shear flow stops $[16,19]$. In the above simulations, shear flows are stopped at $\beta \approx 0.7$. We also conduct simulations when shear flows stop at $\beta \approx 0$ and compare the results at $C a=0.039$.

Time variations of phase angle $\beta$ is illustrated in Fig. 12(a). It can be seen that all capsules recover to resting shapes within a short time. The phase angles for all capsules are almost constant during the whole relaxation process. Particularly for capsules other than the OE 0.28 capsule, their $\beta$ values do not decrease to 0 . This could be limited by the calculation accuracy. Fig. 12(b) shows the contours of biconcave capsules at $\dot{\gamma} t=20$ when shear flows stop at different phase angle. The blue solid line is at $\beta=0.07$ and red dash line at $\beta=0.68$. The dots represent the rim points. Obviously the blue dot is already at the 
(a)

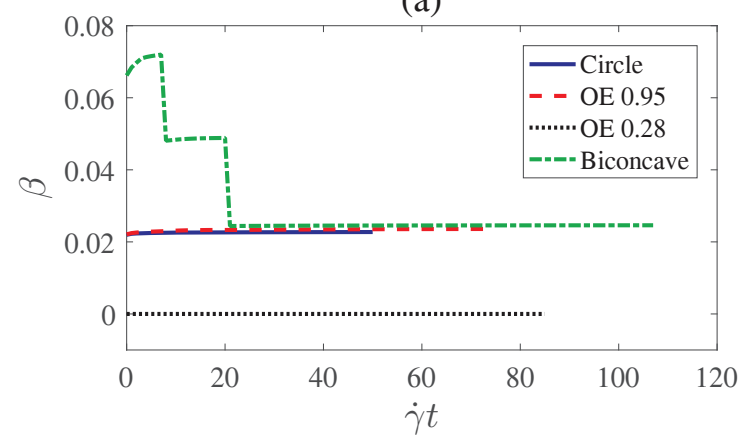

(b)

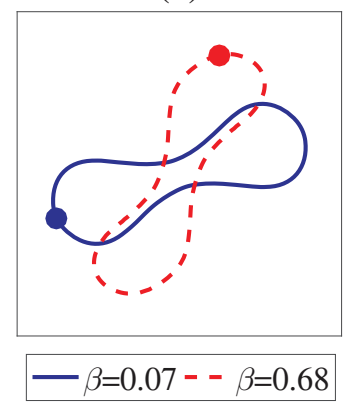

Figure 12: (a) Time variation of phase angle $\beta$ at $C a=0.039$ when shear flows are stopped at $\beta \approx 0$. (b) Contours of biconcave capsules at $\dot{\gamma} t=20$ when shear flows stop at different phase angle. The blue solid line is at $\beta=0.07$ and red dash line at $\beta=0.68$. The dots represent the rim point.

(a)

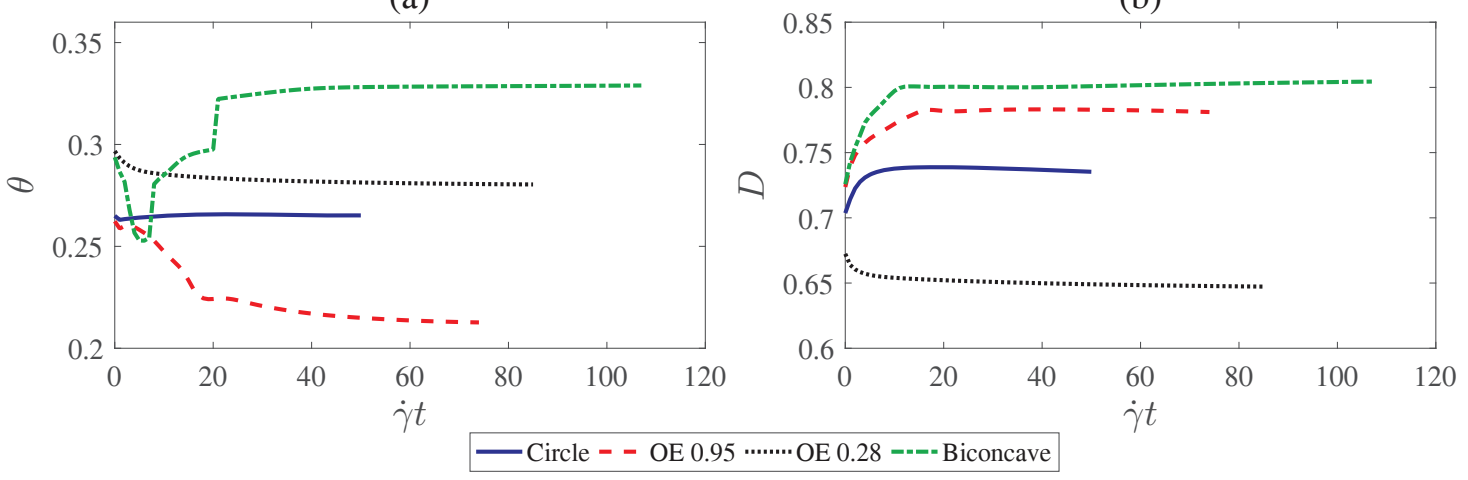

Figure 13: Relaxation from TT in terms of (a) inclination angle $\theta$ and (b) Taylor deformation parameter $D$ at $C a=0.039$. Shear flows are stopped at $\beta \approx 0$.

rim of capsule, while the red dot still needs to tank-treads towards the rim. The shape of blue solid line is the resting shape of biconcave capsule, and the shape of red dash line is deformed from the resting shape.

The inclination angle $\theta$ and Taylor deformation parameter $D$ are shown in Fig. 13. For each capsule, the variation of $\theta$ is within the range of 0.1 . Through the comparison between Figs. 7 and 13(a), the phase angle at which shear flow stops seems to determine the inclination angle after shape relaxation [19]. The $D$ values for OE 0.28 capsule decreases, while the ones for circle, OE 0.95 and biconcave capsules increase. This is because the shapes of all capsules at $\dot{\gamma} t=0$ are similar to their elongated resting shapes. After shear flows stop, the reduction of major axes decreases $D$ values, but the formation of dimples can increase $D$ values. As the resting shape of OE 0.28 capsule has no dimple, the $D$ value is only effected by the decreased major axis. For other three capsules, the decrease of $D$ is compensated by the decrease of minor axes, and eventually the $D$ values increase. 


\section{Conclusion}

In the present work, we have introduced a computational model for simulating the deformation and relaxation of fluid-filled capsules which uses immersed boundary method. The effect of the spontaneous shape on shape memory of capsules is the main focus. For capsules with circle or oblate ellipsoid spontaneous shapes, as long as the reduced area is greater than 0.46 , the resting shapes are still biconcave discoid. However, the dimples are shallower compared to the biconcave capsule. All capsules with noncircular spontaneous shapes show shape memory property. For capsules with circle spontaneous shape, however, the rim points stay as the capsules recover to their resting shapes. Thus the shape memory of membrane may come from noncircular spontaneous shape. Time variations in terms of Taylor deformation parameter, inclination angle and phase angle are also studied and different relaxation modes are compared. The relaxation of bending energy is found to follow two separate modes when bending energy exceeds some critical value. We have posited the range of dimensionless bending energy $\Delta \omega$ and shown its positive relationship with relaxation speed. Our research also indicates that as the oblate ellipsoid spontaneous shape approaches a circle, the relaxation time would increase due to the decrease of $\Delta \omega$. The above results are limited by the 2D model, thus can be used as qualitative reference only. In a $2 \mathrm{D}$ model, the shear elasticity does not play any role for the shape memory. For more complicated capsules like RBCs, a 3D model in combination of both shear elastic and bending anisotropy is required to further study the shape memory and its dependence on membrane properties. Still, this research may help to identify different spontaneous shapes of capsules through future experiments.

\section{Acknowledgments}

We acknowledge the support from the National Natural Science Foundation of China (No. 51505455), the Science Fund for Creative Research Groups of the National Natural Science Foundation of China (No. 51521064) and Applied Research Project of Public Welfare Technology of Zhejiang Province under Grant (No. 2015C31109). We would like to thank Dr. Pengfei Wang for his helpful discussions during this work.

\section{References}

[1] M. Abkarian, M. Faivre and A. Viallat, Swinging of red blood cells under shear flow, Phys Rev Lett, 98 (2007), 188302.

[2] P. Bagchi, Mesoscale simulation of blood flow in small vessels, Biophys J, 92 (2007), 18581877.

[3] D. Barthes-Biesel, A. Diaz and E. Dhenin, Effect of constitutive laws for two-dimensional membranes on flow-induced capsule deformation, Journal of Fluid Mechanics, 460 (2002), 211-222. 
[4] O. K. Baskurt, and H. J. Meiselman, Determination of red blood cell shape recovery time constant in a Couette system by the analysis of light reflectance and ektacytometry, Biorheology, 33 (1996), 489-503.

[5] B. Blais, M. Lassaigne, C. Goniva, L. Fradette and F. Bertrand, A semi-implicit immersed boundary method and its application to viscous mixing, Computers and Chemical Engineering, 85 (2016), 136-146.

[6] N. Cardenas and S. K. Mohanty, Investigation of shape memory of red blood cells using optical tweezers and quantitative phase microscopy, Imaging, Manipulation, and Analysis of Biomolecules, Cells, and Tissues X, 8225 (2012), 82252B.

[7] D. Cordasco, A. Yazdani and P. Bagchi, Comparison of erythrocyte dynamics in shear flow under different stress-free configurations, Physics of Fluids, 26 (2014), 041902.

[8] L. M. Crowl and A. L. Fogelson, Computational model of whole blood exhibiting lateral platelet motion induced by red blood cells, Int J Numer Method Biomed Eng, 26 (2010), 471-487.

[9] L. Crowl and A. L. Fogelson, Analysis of mechanisms for platelet near-wall excess under arterial blood flow conditions, Journal of Fluid Mechanics, 676 (2011), 348-375.

[10] M. Dao, C. Lim, and S. Suresh, Mechanics of the human red blood cell deformed by optical tweezers, Journal of the Mechanics and Physics of Solids, 51 (2003), 2259-2280.

[11] J. Dupire, M. Socol, and A. Viallat, Full dynamics of a red blood cell in shear flow, Proceedings of the National Academy of Sciences of the United States of America, 109 (2012), 20808-20813.

[12] E. Evans and Y. C. Fung, Improved measurements of the erythrocyte geometry, Microvasc Res, 4 (1972), 335-347.

[13] E. Evans and R. Hochmuth, Membrane viscoelasticity, Biophysical Journal, 16 (1976), 1-11.

[14] D. A. Fedosov, M. Dao, G. E. Karniadakis and S. Suresh, Computational biorheology of human blood flow in health and disease, Ann Biomed Eng, 42 (2014), 368-387.

[15] T. M. Fischer, M. Stohr-Lissen and H. Schmid-Schonbein, The red cell as a fluid droplet: Tank tread-like motion of the human erythrocyte membrane in shear flow, Science, 202 (1978), 894-896.

[16] T. Fischer, Shape memory of human red blood cells, Biophysical Journal, 86 (2004), 33043313.

[17] T. M. Fischer, Tank-tread frequency of the red cell membrane: Dependence on the viscosity of the suspending medium, Biophys J, 93 (2007), 2553-2561.

[18] T. M. Fischer and R. Korzeniewski, Threshold shear stress for the transition between tumbling and tank-treading of red blood cells in shear flow: Dependence on the viscosity of the suspending medium, Journal of Fluid Mechanics, 736 (2013), 351-365.

[19] J. Gounley and Y. Peng, Shape recovery of elastic capsules from shear flow induced deformation, Communications in Computational Physics, 16 (2014), 56-74.

[20] W. Helfrich, Elastic properties of lipid bilayers: Theory and possible experiments, Z Naturforsch C, 28 (1973), 693-703.

[21] J. F. Hoffman, Biconcave shape of human red-blood-cell ghosts relies on density differences between the rim and dimple of the ghost's plasma membrane, Proc Natl Acad Sci U S A, 113 (2016), 14847-14851.

[22] B. Kaoui, A. Farutin and C. Misbah, Vesicles under simple shear flow: Elucidating the role of relevant control parameters, Phys Rev E Stat Nonlin Soft Matter Phys, 80 (2009), 061905.

[23] B. Kaoui, J. Harting and C. Misbah, Two-dimensional vesicle dynamics under shear flow: Effect of confinement, Phys Rev E Stat Nonlin Soft Matter Phys, 83 (2011), 066319. 
[24] S. R. Keller and R. Skalak, Motion of a tank-treading ellipsoidal particle in a shear flow, Journal of Fluid Mechanics, 120 (1982), 27-47.

[25] S. Kwak and C. Pozrikidis, Effect of membrane bending stiffness on the axisymmetric deformation of capsules in uniaxial extensional flow, Physics of Fluids, 13 (2001), 1234-1242.

[26] D. V. Le, Effect of bending stiffness on the deformation of liquid capsules enclosed by thin shells in shear flow, Physical Review E, 82 (2010), 016318.

[27] X. Niu, L. Shi, T.-W. Pan and R. Glowinski, Numerical simulation of the motion of inextensible capsules in shear flow under the effect of the natural state, Communications in Computational Physics, 18 (2015), 787-807.

[28] Z. Peng, A. Mashayekh and Q. Zhu, Erythrocyte responses in low-shear-rate flows: Effects of non-biconcave stress-free state in the cytoskeleton, Journal of Fluid Mechanics, 742 (2014), 96-118.

[29] Z. Peng, S. Salehyar and Q. Zhu, Stability of the tank treading modes of erythrocytes and its dependence on cytoskeleton reference states, Journal of Fluid Mechanics, 771 (2015), 449467.

[30] C. S. Peskin, The immersed boundary method, Acta Numerica, 11 (2002), 479-517.

[31] H.-N. Polwaththe-Gallage, S. C. Saha, E. Sauret, R. Flower, and Y. Gu, Numerical investigation of motion and deformation of a single red blood cell in a stenosed capillary, International Journal of Computational Methods, 12 (2015), 1540003.

[32] C. Pozrikidis, Effect of membrane bending stiffness on the deformation of capsules in simple shear flow, Journal of Fluid Mechanics, 440 (2001), 269-291.

[33] K. Sinha and M. D. Graham, Dynamics of a single red blood cell in simple shear flow, Phys Rev E Stat Nonlin Soft Matter Phys, 92 (2015), 042710.

[34] R. Skalak, A. Tozeren, R. P. Zarda and S. Chien, Strain energy function of red blood cell membranes, Biophys J, 13 (1973), 245-264.

[35] J. M. Skotheim and T. W. Secomb, Red blood cells and other nonspherical capsules in shear flow: oscillatory dynamics and the tank-treading-to-tumbling transition, Physical Review Letters, 98 (2007), 078301.

[36] Y. Sui, Y. T. Chew and H. T. Low, A lattice boltzmann study on the large deformation of red blood cells in shear flow, International Journal of Modern Physics C, 18 (2007), 993-1011.

[37] Y. Sui, Y. T. Chew, P. Roy and H. T. Low, Effect of membrane bending stiffness on the deformation of elastic capsules in extensional flow: A lattice boltzmann study, International Journal of Modern Physics C, 18 (2007), 1277-1291.

[38] Y. Sui, Y. T. Chew, P. Roy, X. B. Chen and H. T. Low, Transient deformation of elastic capsules in shear flow: effect of membrane bending stiffness, Physical Review E, 75 (2007), 066301.

[39] Y. Sui, X. B. Chen, Y. T. Chew, P. Roy and H. T. Low, Numerical simulation of capsule deformation in simple shear flow, Computers \& Fluids, 39 (2010), 242-250.

[40] G. Tryggvason, B. Bunner, A. Esmaeeli, D. Juric, N. Al-Rawahi, W. Tauber, J. Han, S. Nas and Y. J. Jan, A front-tracking method for the computations of multiphase flow, Journal of Computational Physics, 169 (2001), 708-759.

[41] J. Walter, A. V. Salsac and D. Barthes-Biesel, Ellipsoidal capsules in simple shear flow: Prolate versus oblate initial shapes, Journal of Fluid Mechanics, 676 (2011), 318-347.

[42] T. Ye, N. Phan-Thien, B. C. Khoo and C. T. Lim, A file of red blood cells in tube flow: A three-dimensional numerical study, Journal of Applied Physics, 116 (2014), 124703. 